\title{
Coupled Pb Chains on Si(557): Origin of One-Dimensional Conductance
}

\author{
C. Tegenkamp, ${ }^{1}$ T. Ohta, ${ }^{2,3}$ J.L. McChesney, ${ }^{2,4}$ H. Dil, ${ }^{3}$ E. Rotenberg, ${ }^{2}$ H. Pfnür, ${ }^{1}$ and K. Horn ${ }^{3}$ \\ ${ }^{1}$ Institut für Festkörperphysik, Leibniz-Universität Hannover, 30167 Hannover, Germany \\ ${ }^{2}$ MS 6-2100, Advanced Light Source, Lawrence Berkeley National Laboratory, Berkeley, California 94720, USA \\ ${ }^{3}$ Fritz-Haber Institut der Max-Planck Gesellschaft, 14195 Berlin, Germany \\ ${ }^{4}$ Montana State University, Bozeman, Montana 53706, USA
}

(Received 31 May 2007; published 22 February 2008)

\begin{abstract}
$\mathrm{The} \mathrm{Pb} / \mathrm{Si}(557)$ system exhibits a strong anisotropy in conductance below $78 \mathrm{~K}$, with the evolution of a characteristic chain structure. Here we show, using angle-resolved photoemission, that chain ordering results in complete Fermi-like nesting in the direction normal to the chains; in addition, the domain structure along the chains forms split-off valence bands with mesoscopic Fermi wavelengths which induce the $1 \mathrm{D}$ conductance without further instabilities at low temperatures.
\end{abstract}

PACS numbers: 73.20.At, 68.65.-k, 73.21. $-\mathrm{b}, 73.25 .+\mathrm{i}$

Confinement of electrons in low-dimensional structures induces intriguing transport phenomena and electronic properties. This scenario of strongly interacting electrons is described within the framework of a Luttinger liquid (LL) rather than by a Fermi liquid (FL) [1]. In an intuitive picture, this changeover is intimately related to the geometry of the structure; i.e., the interaction between electrons is enhanced in low-dimensional systems. An obvious strategy of making one-dimensional (1D) conductors is to use anisotropic solid state bulk materials like Bechgaard salts [2,3] or, as shown recently, $\mathrm{Ca}_{2} \mathrm{Ru}_{2} \mathrm{O}_{7}$ [4,5]. Anisotropic adsorbate structures, realized on (vicinal) surfaces, are even more flexible, because the structure can be controlled on an atomic scale, and the electronic structure can be changed gradually by varying the degree of localization, e.g., by changing terrace widths. Submonolayer coverages of $\mathrm{Ag}$ and $\mathrm{Au}$ on vicinal $\mathrm{Si}(111)$ surfaces form chain structures showing indeed localized states perpendicular to the chain direction [6-8]. However, these weakly interacting systems often undergo instabilities, e.g., Peierls- or Mott-Hubbard driven phase transitions into insulating states at low temperature (see, e.g., [9]), and the 1D character of charge carriers is rarely established in transport measurements.

$\mathrm{The} \mathrm{Pb} / \mathrm{Si}(557)$ system is unique in this respect since macroscopic conductance measurements $[10,11]$ reveal a slightly anisotropic temperature activated conductance above $T_{c}=78 \mathrm{~K}$. Below this temperature the conductance switches reversibly to quasi-1D and metallic along the step direction, while in the perpendicular direction conductance is indistinguishable from the residual conductance of the substrate. In contrast to all systems exhibiting quasi 1Dconductance properties investigated so far, this system turns into a quasi-1D metallic conductance state at low temperatures with no indications of further instabilities.

This intriguing behavior requires an explanation in terms of the interplay of the geometric and electronic structure across the phase transition. Here using angleresolved photoemission (ARPES), we demonstrate that the interplay of the overlayer structure and the substrate leads to an electronically stabilized arrangement of chain structures and has direct consequences on the formation and filling of electronic bands that results in the observed transport properties. In particular, we demonstrate 1D conductance is a direct consequence of the two-dimensional interactions between the chains and the terraces thus avoiding to a large extent the instabilities of purely $1 \mathrm{D}$ systems.

As shown by scanning tunneling microscopy (STM) and detailed analysis of the data, the Pb-covered surface is restructured by an annealing process into large $\mathrm{Pb}$-covered mini terraces with a width of $d=1.55 \pm 0.05 \mathrm{~nm}$, forming a chainlike structure that is $4 \frac{2}{3} \mathrm{Si}$ chains wide and is separated by double steps of $0.314 \mathrm{~nm}$ height, i.e., the surface is refacetted into a (223) structure [12]. Below $78 \mathrm{~K}$, the step structure is well ordered, and the chain structure along the terraces exhibits a tenfold superperiodicity [11]. Low energy electron diffraction (LEED) reveals that the local $\mathrm{Pb}$-induced order corresponds to a

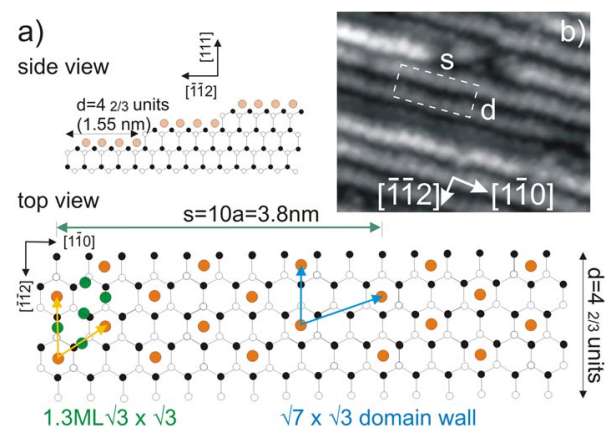

FIG. 1 (color online). (a) Top-view and side-view model of the wire structure with 1.3 ML of Pb obtained from STM and LEED measurements. The light gray (orange) circles denote $\mathrm{Pb}$ atoms on $\mathrm{H} 3$ positions. The full monolayer structure is shown only for the first $\sqrt{3} \times \sqrt{3}$ unit cell. (b) STM of the Pb wires $(T=40 \mathrm{~K})$. The interchain distance $d$ is found to be $1.5 \pm 0.1 \mathrm{~nm}$, whereas along the wire structure a tenfold periodicity $s=3.8 \mathrm{~nm}$ can be observed [11-13]. 
$\sqrt{3} \times \sqrt{3} R 30^{\circ}$ structure, which is split due to the formation of regularly spaced domain walls yielding the tenfold superperiodicity $[12,13]$. Since a uniformly stepped surface with the (557) orientation would require $1.9 \mathrm{~nm}$ wide terraces, the narrower chainlike structures must be stabilized by $\mathrm{Pb}$. A structural model of the ordered phase is shown in Fig. 1 together with a section of an STM image; the structural unit cell is marked by dashed lines. Both the superperiodicity and the long range order in the step-step distance are destroyed when going through the sharp and reversible first order phase transition above $78 \mathrm{~K}$. In this Letter we demonstrate how the electronic structure in the vicinity of the Fermi level induces this behavior.

The $\mathrm{Si}(557)$ sample was cleaned in ultrahigh vacuum (base pressure $3 \times 10^{-11} \mathrm{mbar}$ ) by the standard procedures described in Ref. [10]. The $\mathrm{Pb}$ monolayer (ML) was prepared by $\mathrm{Pb}$ evaporation at $T \leq 100 \mathrm{~K}$ followed by annealing to $640 \mathrm{~K}$ for several minutes to ensure full multilayer desorption and rearrangement of the step structure. After preparation, the sample was transferred onto a $\mathrm{He}$-cooled manipulator, and the resulting $\mathrm{Pb}$ structure was checked by LEED at temperatures around $40 \mathrm{~K}$. For the layers measured in detail with ARPES we always saw the expected regular step structure described above, as well as the correct spot splitting of the diffuse $\sqrt{3} \times \sqrt{3}$ structure, indicating tenfold periodicity along the chains. ARPES measurements were carried out at beam line 7.0.1 at Advanced Light Source, using a computer-controlled setup for polar and azimuthal scans in $k$ space. The photoelectrons were detected with a SCIENTA R4000 spectrometer. The angular acceptance was about $0.15^{\circ}$ for each data point. The sample was oriented so that the polarization plane of the light was parallel to [11̄0] direction. An optimal cross section for the $\mathrm{Pb}$ structures in spectroscopy was obtained for $h \nu=160 \mathrm{eV}$.

For the clean $\mathrm{Si}(557)$ the photoemission data of previous studies $[14,15]$ were well reproduced. They are dominated by the features of the $7 \times 7$ reconstructed (111)-oriented mini terraces. The characteristic surface states and their strong intensity dependence as a function of momentum were used to calibrate the surface Brillouin zones (SBZ) with respect to the (111) unit cell. The valence band maximum, $E_{\mathrm{VBM}}$ for the clean substrate is around $0.65 \mathrm{eV}$ below the Fermi energy $E_{F}$.

After preparation of the $\mathrm{Pb}$ chain structure, strongly modified surface states are formed by the interaction of the $\mathrm{Pb}$ monolayer with $\mathrm{Si}$. Figures 2(a) and 2(b) show band dispersions taken along and perpendicular to the wires, respectively [cf. with dotted lines in Fig. 2(c)]. The bands near $E_{F}$ are completely determined by the $\mathrm{Pb}$ modified surface states since $E_{\mathrm{VBM}}$ [see center of Fig. 2(a)] is still $0.2 \mathrm{eV}$ below $E_{F}$. Therefore, electronic transport in these metallic chains is a pure surface property with charge depletion in the adjacent Si bulk material. These scans also clearly demonstrate the two-dimensional coupling in this system. The energy bands disperse in both directions
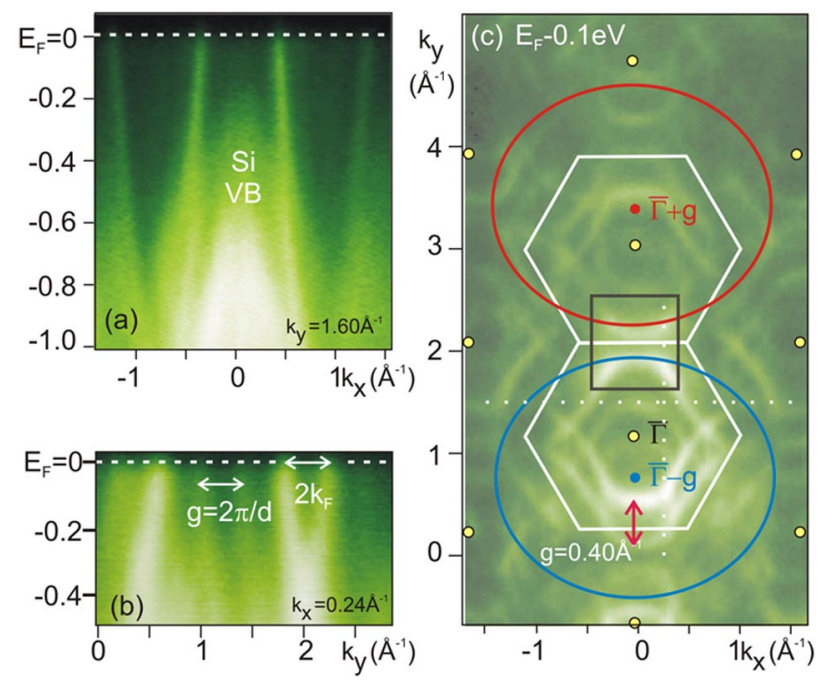

FIG. 2 (color online). (a), (b) Energy dispersion curves of the 1.3 $\mathrm{ML} \mathrm{Pb}$ structure $\left(T_{s}=40 \mathrm{~K}\right.$, high intensity in white) in $k_{x}$ and $k_{y}$ directions along the dotted lines marked in (c). Here the DOS in the $\left(k_{x}, k_{y}\right)$ plane, as measured by ARPES, is plotted at constant energy $E_{F}-0.1 \mathrm{eV}$. In (b), complete filling of the topmost band is demonstrated by the coincidence of the reciprocal lattice vector $g=\frac{2 \pi}{d}$ ( $d$ is the interchain distance) with $2 k_{F}$ at $E_{F}$. Further away from $E_{F}$, as shown in (c), a set of ellipses centered at the $\bar{\Gamma}$ points of the (111) terraces and at centers shifted by $g$ describes most of the intensity distribution. Deviations leading to Fermi nesting are best visible within the square at the center (see Fig. 3).

so that 1D conductance properties are not obvious, but are due to a special structural and electronic coincidence, as explained below.

The intensity distribution in $k$ space $0.1 \mathrm{eV}$ below $E_{F}$, plotted in Fig. 2(c), has a free-electron-like character: it can be reconstructed by ellipses (rather than circles) around the $\bar{\Gamma}$ points of the (111) terraces and, in addition,

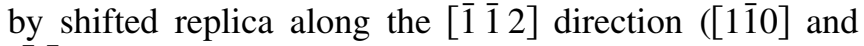
$\left[\begin{array}{lll}\overline{1} & 1 & 2\end{array}\right]$ directions are called $x$ and $y$ directions, respectively, in what follows). The shift vector, $g$, corresponds exactly to the reciprocal lattice vector $g=\frac{2 \pi}{d}=0.40 \pm 0.01 \AA^{-1}$, where $d=1.55 \mathrm{~nm}$ is the interchain distance. Such repeated structures also have been found for a variety of vicinal metallic surfaces [16,17]. For better orientation we marked the $\bar{\Gamma}$ points and the SBZ of the (111) surface in Fig. 2(c).

The fact that ellipses and not circles are found is readily explained by the influence of the terraces: the ratio of the principal axis is $a / b=0.85$, which agrees with $a+g=$ $b$, where $g=\frac{2 \pi}{d}$. This anisotropy correlates qualitatively with the anisotropy found in conductivity above $T_{c}$ [11]. The area of the ellipses is approximately 1.8 times the area of the first (111) SBZ, which is in reasonable agreement with the valence state of $\mathrm{Pb}$, taking into account that the $\mathrm{Pb}(111)$ unit cell is compressed by $20 \%$ compared to $\mathrm{Si}(111)$ due to the mismatch. 

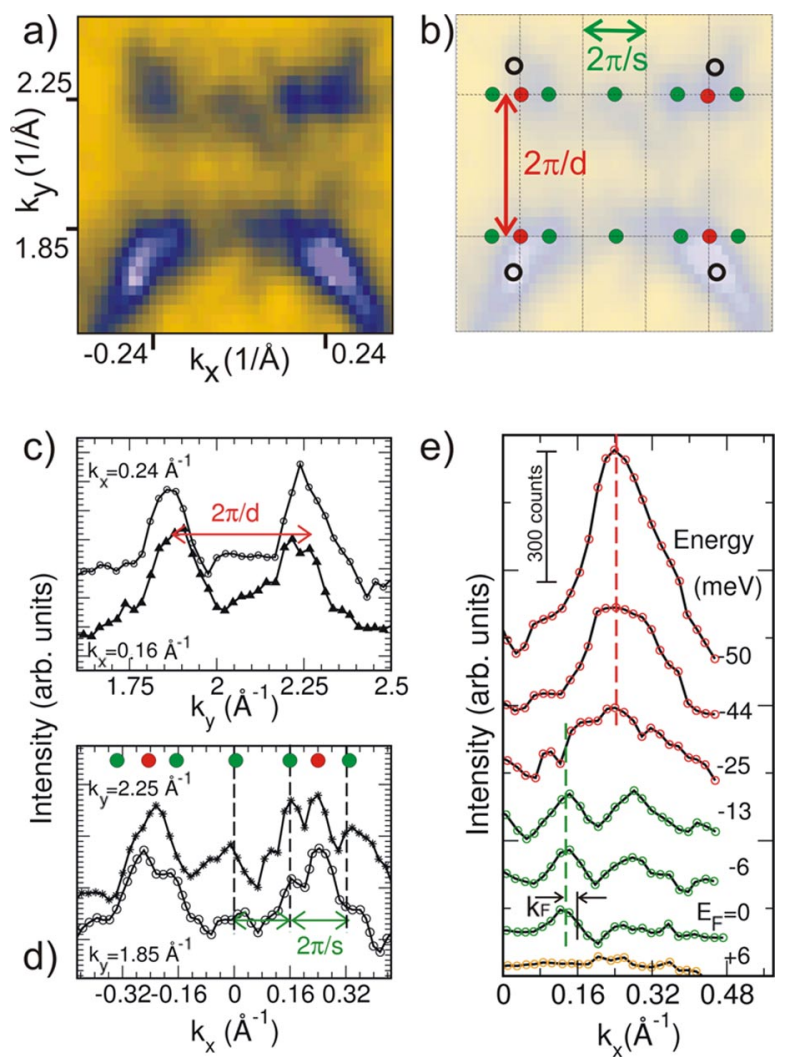

FIG. 3 (color online). (a) Constant energy plot, but at $E_{F}-0.03 \mathrm{eV}$, of the section marked by a square in Fig. 2(c) (low intensity in yellow). (b) Schematic drawing of the nesting points. The rectangles show the SBZ of the $\mathrm{Pb}$-wire structure. (c), (d) Momentum distribution curves (MDCs) from (a) taken along $k_{y}$ and $k_{x}$, respectively, reflecting the interchain distance $d$ and the tenfold periodicity $s$. (e) Sequence of MDC curves along $k_{x}$ at $k_{y}=2.25 \AA^{-1}$ at different energies around $E_{F} \cdot T_{s}=40 \mathrm{~K}$.

However, in order to explain the origin of 1D conductance in this $2 \mathrm{D}$ coupled system at low temperatures, deviations from the free-electron-like picture are necessary. This becomes visible only closer to $E_{F}$ near the SBZ boundaries, as estimated from the phase transition temperature of $78 \mathrm{~K}$. Indeed, such deviations become obvious at $E_{F}-0.03 \mathrm{eV}$ : in Fig. 3(a) we plotted the section of Fig. 2(c) marked by a square, where the complete Fermi surface can be observed due to Umklapp scattering by $+g$ and $-g$. This is accompanied by high intensities, which is important, since the photoemission process is dominated by the local atomic environment, and long range order is reflected only by intensity modulations. In order to facilitate interpretation, the SBZ grid was superimposed onto Fig. 3(a), as shown in Fig. 3(b). It was determined from the geometrical structure found in STM and (independent) LEED measurements (see Fig. 1 and [12]) with the unit vectors $g$ and $f=2 \pi / s=0.16 \AA^{-1}$ (with the tenfold periodicity $s$ ) in the [ $\left.\begin{array}{lll}1 & 1 & 2\end{array}\right]$ and [ $\left.11 \overline{1} 0\right]$ directions, respectively. As origin we took the central $\bar{\Gamma}_{1 \times 1}$ point without further shifts.

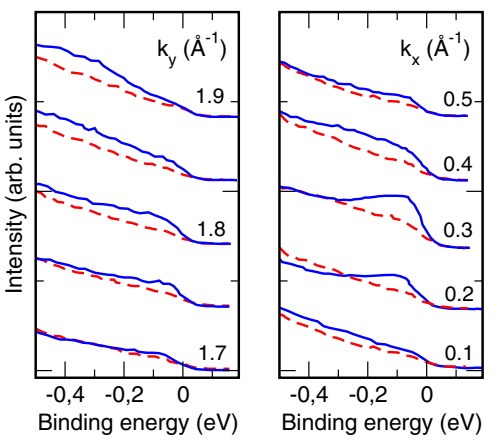

FIG. 4 (color online). Energy distribution curves taken along $k_{y}$ at $k_{x}=0 \AA^{-1}$ (left) and along $k_{x}$ at $k_{y}=1.8 \AA^{-1}$, respectively, below $\left[T_{s}=40 \mathrm{~K}\right.$, solid (blue) line $]$ and above the phase transition $\left[T_{s}=100 \mathrm{~K}\right.$, dashed (red) line].

As shown in Fig. 3(a), the Fermi surface is no longer free-electron-like, but consists of only a few points in $k$ space aligned along lines of constant $k_{y}$ instead of sections of ellipses. The intensity close to the open circles of Fig. 3(b) is the result of an intersection of three ellipses, i.e., does not contain information of long range order and is therefore not considered here. The separation between these lines corresponds to exactly the reciprocal lattice vector $g$, and the intensity is concentrated at the zone boundary in the $k_{y}$ direction, i.e., the Fermi surface is nested. This fact is emphasized even more clearly by looking at the MDC taken at $k_{x}=0.24 \AA^{-1}$ shown in Fig. 3(c). A corresponding energy band is shown in Fig. 2(b) along $k_{y}$. All three plots demonstrate that the Fermi wave vector in [ [ $\left.\begin{array}{lll}1 & 1 & 2\end{array}\right]$ direction fulfills the condition $2 k_{F}=g$. I.e., the uppermost band is completely filled for this direction and a standing wave is formed. In terms of transport, this induces insulating behavior.

Although an opening of a fundamental gap at $E_{F}$ is not expected for a system with quasi one-dimensional conductance, the signature of nesting is manifested by a concentration of DOS at those $k$ points just described, as exemplarily demonstrated by the EDCs in Fig. 4, which is extended over a few tenths of an $\mathrm{eV}$ away from $E_{F}$ at temperatures below the phase transition. Above $T_{c}$, this enhancement at $E_{F}$ has vanished, i.e., the intensity distribution becomes more uniform in $k$ space (data sets drawn in red in Fig. 4). As will be argued below, this effect is induced by structural changes and causes the switching in conductivity.

We now analyze the Fermi surface in more detail in order to determine the nesting conditions effective in this system. This is done by plotting the intensity as a function of $k_{x}$ at constant energy $E_{F}-0.03 \mathrm{eV}$ and constant $k_{y}$, which fulfill the $2 k_{F}=g$ condition, i.e., $k_{y}=1.85 \AA^{-1}$ and $k_{y}=2.25 \AA^{-1}$ in Fig. 3(d), and at different energies around $E_{F}$ in Fig. 3(e). Two characteristic periodic intensity distributions were found in such plots: in addition to the intensity marked by the red circles, which is concen- 
trated in the corners of the SBZ defined by the modulation along the wires (cf. Fig. 1 and [12]) a phase shifted modulation, again with the period $f=2 \pi / s=0.16 \AA^{-1}$, close to the center of the SBZ, is seen. The tenfold periodicity along the chains obviously leads to the formation of a doublet of split bands of the parabolae in $k_{x}$ direction.

The peaks marked by red circles are at the SBZ boundary in both directions. Following the arguments from above, this part of the split band must be exactly filled in both directions and a band gap should be visible for this part. This is what we see indeed in Fig. 3(e). Here intensity plots along the $k_{x}$ direction at $k_{y}=2.25 \AA^{-1}$ are shown at energies between $E_{F}-0.05 \mathrm{eV}$ and $E_{F}$. Starting around $50 \mathrm{meV}$ below $E_{F}$, the intensity at $k_{x}=0.24 \AA^{-1}$, marked by red circles in Fig. 3(b) and 3(d), decreases gradually and vanishes completely at $E_{F}$. In contrast, the satellite peaks (green circles), which appear in Fig. 3(e) around $k_{x}=$ $0.16 \AA^{-1}$, have a finite intensity at $E_{F}$, i.e., these bands cross the Fermi level and are the only ones that contribute to electron transport in the [1 10$]$ direction.

The remarkable insensitivity of $\mathrm{dc}$ conductance to atomic defects in the $\mathrm{Pb}$ chains found in experiment [11] can be explained by the extremely long Fermi wavelength of the conducting band found here. Taking the SBZ with the tenfold periodicity, as done in Fig. 3(b), it turns out that the peaks marked by the green dots are close to the $\bar{\Gamma}$ points, i.e., the Fermi wavelength of these states is above $20 \mathrm{~nm}$ [cf. Fig. 3(e)]. Therefore, the electrons scatter only weakly at defects of atomic size in this ordered state, in agreement with the experimental findings [11].

While the origin of this band splitting close to $E_{F}$ still remains to be clarified (it may be induced directly by the domain structure of the tenfold modulated structure), we can estimate the gap size for the insulating band from Fig. 3(e) to be around $20 \mathrm{meV}$. Since this band has the dominant intensity compared with the conducting band, the formation of this band gap close to $E_{F}$ effectively lowers the total energy of the ordered structure. Therefore, the terrace structure with its particular terrace width as sketched in Fig. 1 appears to be stabilized laterally by an effect with a strong electronic component, similar to the vertical stabilization of so-called magic heights of $\mathrm{Pb}$ islands on $\mathrm{Si}(111)[18,19]$. Moreover, the gap size of $20 \mathrm{meV}$ is of the expected order of magnitude for a critical ordering temperature of $78 \mathrm{~K}$.

Increasing the substrate temperature above $T_{c}=78 \mathrm{~K}$, a structural first order phase transition with abrupt changes in conductance has been found [11]. At $T_{c}$, the dc conductance switches into a $2 \mathrm{D}$ regime, where transport is activated in both directions. A recent detailed structural analysis of this phase transition carried out by our group [20] revealed that this phase transition is commensurateincommensurate coupled with increasing disorder in the system. Both the change in periodicity and the disorder cause removal of the nesting condition in the $\left[\begin{array}{lll}1 & 1 & 2\end{array}\right]$ direc- tion and thus a breakdown of the band filling rule in this direction. This is clearly seen in Fig. 4. The EDC curves at $100 \mathrm{~K}$, shown are just examples, demonstrate a more or less uniform distribution of the DOS close to $E_{F}$ in $k$ space. Correspondingly, the $\mathrm{Pb}$ chains become two-dimensionally conducting above $T_{c}$, as observed [10,11]. The repeated zone structures are still visible in ARPES (not shown), but with the slightly smaller $g$ vectors, as also found in our structural studies [20].

Summarizing, we have shown by ARPES that monolayer stripes of $\mathrm{Pb}$ on $\mathrm{Si}(557)$ are two-dimensionally coupled. However, $\mathrm{Pb}$ induces periodic self-organization of the terrace structure below $78 \mathrm{~K}$ that deviates from the macroscopic orientation and may in part be electronically stabilized. As seen here, this process results in twodimensional nesting conditions at the Fermi surface with exact band filling in the $\left[\begin{array}{lll}\overline{1} & 1 & 2\end{array}\right]$ direction normal to the step structure. In the direction along the chains, a doublet of bands is found at $E_{F}$, with only one of them conducting. The long superperiodicity along the chains causes a quasimesoscopic Fermi wavelength. Since these conditions are stabilized by a 2D mechanism, no further instabilities are expected at low temperature, and the material remains an almost ideal conductor.

We thank A. Bostwick for experimental support and fruitful discussions. This work is supported by the DFG, by the Max Planck Society and the ESF (T. O., K. H.), and by DOE and Department of Defense EPSCoR grants (J.L.M.). A.L.S. is supported by the Director, Office of Science, Office of Basic Energy Sciences, of the DOE (No. DE-AC02-05CH11231).

[1] J. Voit, Rep. Prog. Phys. 58, 977 (1995).

[2] S. Roth and C. Caroll, One-Dimensional Metals (WileyVCH, Weinheim, 2004).

[3] R. Claessen et al., Phys. Rev. Lett. 88, 096402 (2002).

[4] F. Baumberger et al., Phys. Rev. Lett. 96, 107601 (2006).

[5] E. Ohmichi et al., Phys. Rev. B 70, 104414 (2004).

[6] J. R. Ahn et al., Phys. Rev. Lett. 91, 196403 (2003).

[7] J. N. Crain et al., Phys. Rev. Lett. 90, 176805 (2003).

[8] J. N. Crain and F. J. Himpsel, Appl. Phys. A 82, 431 (2006).

[9] J. R. Ahn et al., Phys. Rev. Lett. 93, 106401 (2004).

[10] C. Tegenkamp et al., Phys. Rev. Lett. 95, 176804 (2005).

[11] C. Tegenkamp et al., Eur. Phys. J. B 43, 557 (2005).

[12] M. Czubanowski et al., New J. Phys. 9, 338 (2007).

[13] C. Tegenkamp and H. Pfnür, Surf. Sci. 601, 2641 (2007).

[14] R. Losio et al., Phys. Rev. B 61, 10845 (2000).

[15] J. E. Northrup, Phys. Rev. Lett. 57, 154 (1986).

[16] A. Mugarza and J. E. Ortega, J. Phys. Condens. Matter 15, S3281 (2003).

[17] J. E. Ortega et al., New J. Phys. 7, 101 (2005).

[18] G. Wang et al., Surf. Sci. 551, 151 (2004).

[19] K. Budde et al., Phys. Rev. B 61, R10602 (2000).

[20] M. Czubanowski and C. Tegenkamp et al. (unpublished). 